6. J.E. Goell, A circular harmonic computer analysis of rectangular dielectric waveguides, Bell Syst Tech J 48 (1969), 2133-2160.

7. M.P.S. Rao, B. Prasad, P. Khastgir, and S.P. Ojha, Theoretical study of thin infrared filter with alternate concentric regions of dielectric and partially conducting materials, Photon Optoelectron 2 (1994), 157-167.

8. M.P.S. Rao, B. Prasad, P. Khastgir, and S.P. Ojha, Modal cutoff conditions for an optical waveguide with a hypocycloidal cross section, Microwave Opt Technol Lett 14 (1997), 177-180.

9. P. Sharan, P. Khastgir, S.P. Ojha, and B. Prasad, Metal-clad optical waveguide with a cordiod shaped cross-section and their modal characteristics, Microwave Opt Technol Lett 16 (1997), 271-275.

10. M.P.S. Rao, V. Singh, B. Prasad, and S.P. Ojha, Modal cutoff conditions of a hypocycloidal waveguide with various types of metal loading on the core boundaries, Microwave Opt Technol Lett 19 (1998), 152-158.

11. M.P.S. Rao, V. Singh, B. Prasad, and S.P. Ojha, Weak guidance and strong guidance studies of the dispersion curves of a waveguide with a hypocycloidal cross-section, Photon Optoelectron 5 (1998).

12. M.P.S. Rao, V. Singh, B. Prasad, and S.P. Ojha, Modal characteristics and dispersion curves of a hypocycloidal optical waveguide, Optik 110 (1999), 81-85.

13. B. Prasad, M.P.S. Rao, V. Singh, S.P. Ojha, Modal characteristics and cutoff conditions of a hypocycloidal waveguide with chiral media in the core and the cladding, Optik (1999, in press).

14. V. Singh, S.P. Ojha, B. Prasad, and L.K. Singh, Optical and microwave dispersion curves of an optical waveguide with a guiding region having a cross-section with a lunar shape, Optik (1999).

15. V. Singh, S.P. Ojha, and B. Prasad, Modal dispersion characteristics of a metal-clad optical waveguide with a guiding cross-section resembling a lens, Photon Optoelectron (1999, in press).

16. V. Singh, S.P. Ojha, and L.K. Singh, Numerically obtained dispersion curves of a waveguide having a cross-sectional shape resembling a lune with various types of metal loading on the core boundaries, Int Conf Fiber Opt Photon, India, 1998, vol. 1, pp. 279-282.

17. V. Singh, S.P. Ojha, and L.K. Singh, Metal-clad optical waveguide having lens-shaped cross-sections and their dispersion curves, Nat Symp Antennas Propagat, India, 1998, vol. 1, pp. 188-191.

18. V.N. Mishra, V. Singh, B. Prasad, and S.P. Ojha, Weak guidance modal dispersion curves of an optical waveguide having a double convex lens cross-section, Microwave Opt Technol Lett (1999, in press).

19. M.P.S. Rao, V. Singh, B. Prasad, and S.P. Ojha, An analytical study of the dispersion curves of an annular waveguide made of liquid crystal, Photon Optoelectron 5 (1998), 73-88.

20. V. Singh, S.P. Ojha, and L.K. Singh, Modal behavior, cutoff condition and dispersion characteristics of an optical waveguide with a core cross-section bounded by two spirals, Microwave Opt Technol Lett 21 (1999), 121-124.

21. V. Singh, B. Prasad, and S.P. Ojha, An analytical study of the cutoff conditions and the dispersion curves of a waveguide with a cross-sectional shape resembling an ellipse compressed along the minor axis, Microwave Opt Technol Lett (1999, in press).

(C) 1999 John Wiley \& Sons, Inc.

CCC 0895-2477/99

\section{PERFECTLY MATCHED LAYER TERMINATION FOR FINITE-ELEMENT MESHES: IMPLEMENTATION AND APPLICATION}

\author{
Youssry Y. Botros ${ }^{1}$ and John L. Volakis ${ }^{1}$ \\ ${ }^{1}$ Radiation Laboratory \\ Department of Electrical Engineering and Computer Science \\ University of Michigan \\ Ann Arbor, Michigan 48109-2122
}

Received 11 May 1999

ABSTRACT: Perfectly matched layer (PML) absorbers deteriorate the condition of the resulting finite-element sparse systems. Therefore, poor convergence scenarios are observed when an iterative solver is employed. In this work, we show that, by choosing the PML parameters in an optimal manner, substantial speedup in the solution convergence is achieved without affecting PML absorption. A robust preconditioned solver with nearly no breakdown possibilities is suggested, implemented, and tested for two microwave circuit applications. (c) 1999 John Wiley \& Sons, Inc. Microwave Opt Technol Lett 23: 166-172, 1999.

Key words: finite-element methods; matched layer termination; microstrip line; spiral inductor

\section{INTRODUCTION}

When introduced by Sacks et al. [1], the perfectly matched layer (PML) absorber was considered a novel, efficient, and reliable way to terminate finite-element (FE) computational domains. They offer ease of implementation coupled with excellent absorption characteristics [2]. Unlike absorbing boundary conditions (ABCs) [3, 4], PML truncation schemes do not require a priori knowledge of propagation constants within the computational domain, and the use of boundary derivatives is avoided altogether. Also, PML termination schemes facilitate de-embedding and parameter extraction [5]. Because of these advantages, PML absorbers have been extensively employed for truncating FEM domains [6, 7]. However, PML absorbers represent active anisotropic materials, and their inclusion within the computational domain significantly deteriorates the condition of the resulting FEM systems. Consequently, PML-truncated meshes have the undesirable property of being slow to converge [8].

Two steps are proposed for improving solution convergence. First, an optimal selection of the PML parameters is given, providing a compromise between convergence and absorption. Second, a robust iterative solver is proposed to solve sparse FEM systems generated when the computational domain is terminated by the PML. Using the designed PML absorbers, we proceed to use them in truncating computational domains for microwave circuit analysis. Two examples are considered: one deals with feed and input impedance characterization of microstrip lines, and the other considers analysis of a spiral inductor with an air bridge.

\section{PML PARAMETERS}

Consider a wave incident upon the interface between two media as shown in Figure 1. Layer 2 is a uniaxial absorber with $\overline{\bar{\mu}}_{r}$ and $\overline{\bar{\epsilon}}_{r}$ representing its relative constitutive parameter tensors

$$
\overline{\bar{\mu}}_{r}=\overline{\bar{\epsilon}}_{r}=\left(\begin{array}{ccc}
a_{2} & 0 & 0 \\
0 & b_{2} & 0 \\
0 & 0 & c_{2}
\end{array}\right) .
$$




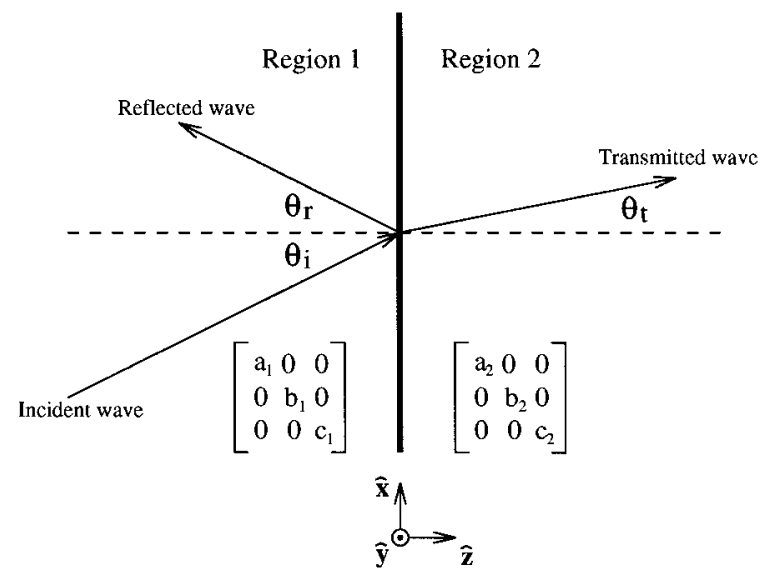

Figure 1 Plane-wave incidence on an interface between two diagonally anisotropic half-spaces

According to [1], the interface reflection coefficients vanish by choosing $a_{2}=b_{2}=1 / c_{2}=\alpha-j \beta$, where $\alpha$ represents the phase shift factor and $\beta$ is the attenuation coefficient. The convergence of FEM systems with PML truncation will be examined or characterized using the parameter $r$, given by

$$
r=\frac{\text { number of iterations before convergence }}{\text { FEM system size }} .
$$

For small values of $r$, the system is rapidly converging. Correspondingly, the system is poorly converging when $r$ approaches unity.

\section{EFFECTS OF $\alpha$ AND $\beta$ ON CONVERGENCE}

Extensive studies have been performed to characterize the PML performance when used to terminate finite-element domains (see, for example, $[7,9,10]$ ). These studies indicated that $\alpha$ plays no role in the absorption, and for maximum absorption, $\beta$ was selected near unity. The effect of $\alpha$ and $\beta$ on the convergence of iterative schemes was tested in [11],

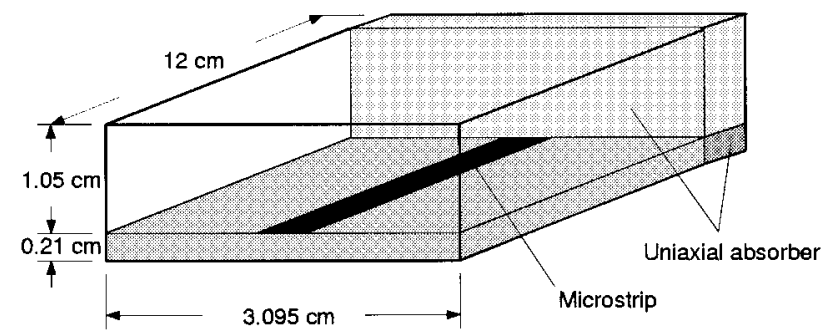

Figure 2 Microstrip-line geometry with PML truncation

where a PML layer was employed to terminate a domain within a waveguide. In [11], both convergence and absorption were studied separately. However, to generate a set of useful design curves for selecting the PML parameters, convergence should be studied in conjunction with absorption, and this study is the first goal of the present paper. We will also consider a wider range of both parameters than that discussed in [11].

Let us consider a simple microstrip line terminated with a PML layer as shown in Figure 2. To evaluate the role of $\alpha$ and $\beta$ in absorption and convergence, both the coefficient of the reflected wave at the microstrip line $R$ and the convergence parameter $r$ are extracted after executing the FEM solution as $\alpha$ and $\beta$ are varied.

Figure 3 displays the PML performance as $\alpha$ is varied with $\beta$ kept at unity to achieve maximum absorption $[9,10]$. It is clear that convergence is substantially improved for $\alpha$ greater than or equal to unity, but absorption was not as greatly affected by changes in $\alpha$ (for $\alpha \leq 2.5$ ). Figure 4 displays both absorption and convergence as functions of $\beta$. We now observe that convergence deteriorates with increasing $\beta$. This is due to abrupt changes in the field values among adjacent numerical cells as the attenuation coefficient $\beta$ is increased. It is also seen that $\beta$ should be selected equal to unity to achieve maximal attenuation.

From Figures 3 and 4, it can be concluded that, with $\beta=1, \alpha$ can be chosen as 0,1 , or 2.25 for maximum absorption. The case of $\alpha=0$ leads to poor convergence of
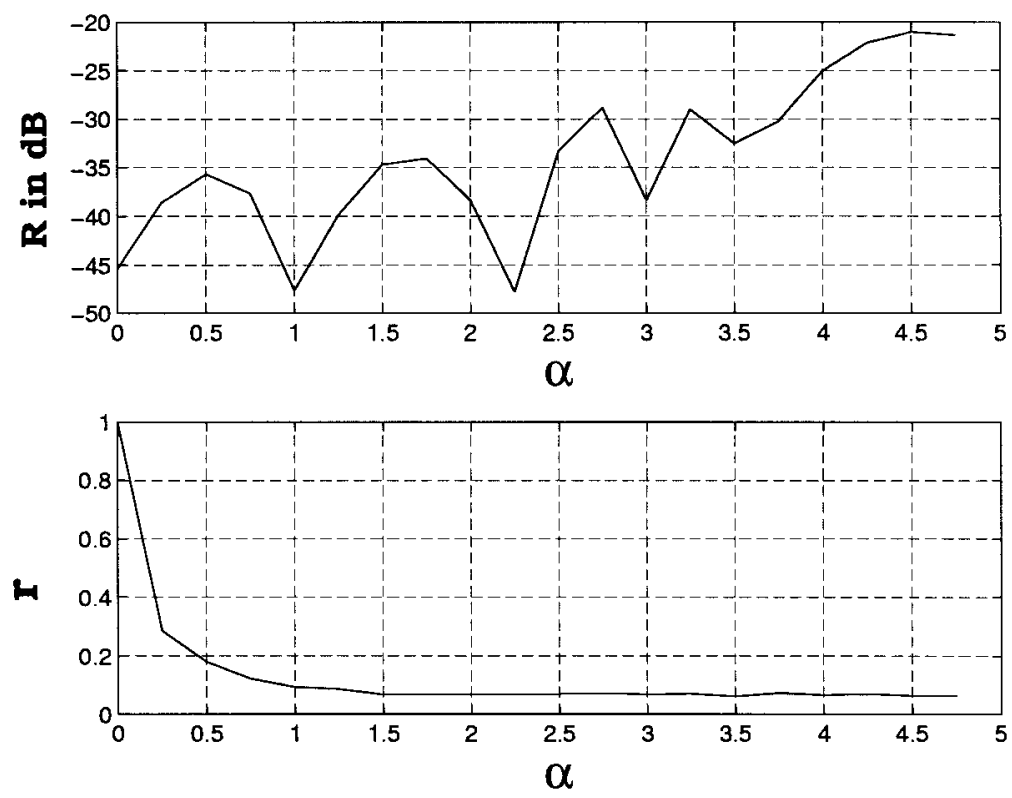

Figure 3 Absorption level (in decibels) and convergence factor as functions of $\alpha$ 

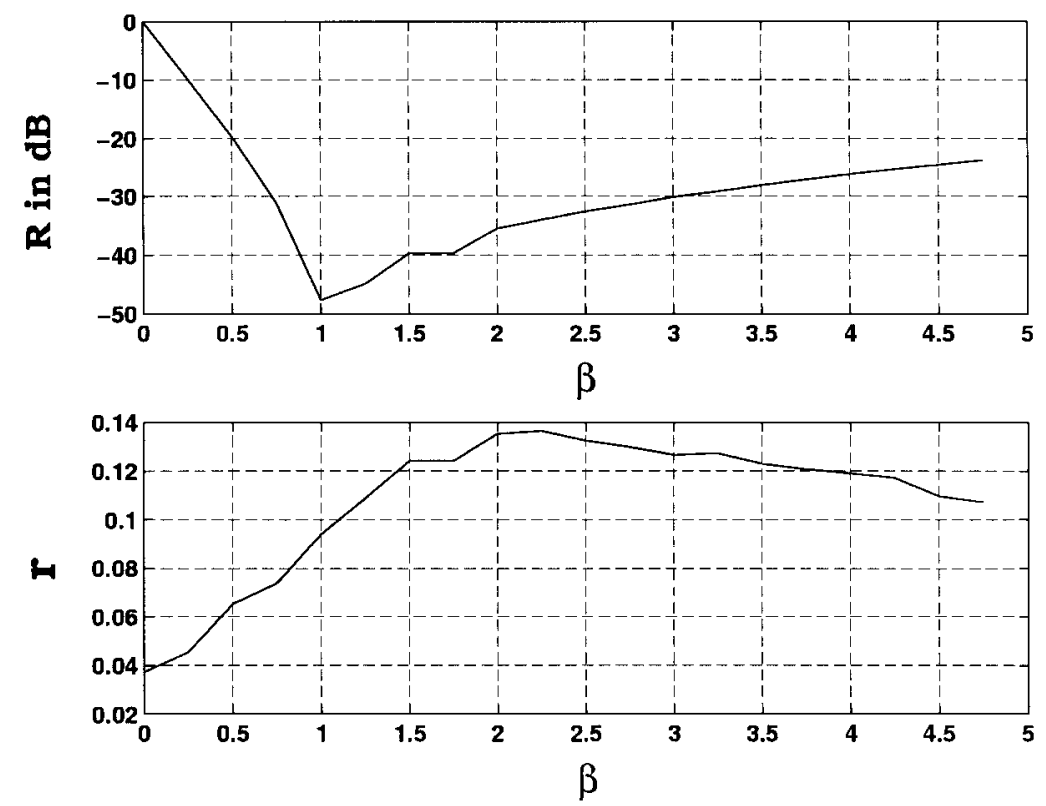

Figure 4 Absorption level (in decibels) and convergence factor as functions of $\beta$

the iterative solver, as displayed in Figure 3. Also, for $\alpha \leq 1$, the PML material is associated with a nonphysical wave speed greater than that of light. This results in a considerable deterioration in the matrix condition, and consequently, the iterative solver convergence. Further, several tests for planar structures demonstrated that the optimal value of the phase parameter $\alpha$ is unity $[8,11]$.

From the above, we conclude that the recommended PML parameters are $\alpha=\beta=1$, corresponding to a wavenumber of $k_{0}=\left(2 \pi / \lambda_{0}\right)$, where $\lambda_{0}$ is the free-space wavelength. This selection achieves maximum attenuation for incoming waves without deteriorating the iterative solver convergence.

\section{GMRES SOLVER}

Three different solvers were implemented and tested for comparison purposes. The first is the biconjugate (BCG) gradient, which has low CPU time per iteration and minimal memory cost. However, it lacks robustness, and does not guarantee convergence, especially for poorly conditioned systems. Also, it has irregular convergence characteristics with two breakdown possibilities [12, 13]. The quasiminimal residue (QMR) solver has better convergence features and lower breakdown or divergence possibilities. Nevertheless, for a given system, both BCG and QMR converge nearly in the same number of iterations, with QMR having the better error history. On the other hand, the generalized minimal residual (GMRES) solver is robust, and guarantees convergence with no breakdown possibilities. It leads to the smallest error among all solvers for a fixed number of matrix vector products $[8,14]$. Also, it can be optimized by changing the number of search vectors per restart $m$ and/or by introducing various preconditioning schemes within the iteration loop [12]. Figure 5 shows a comparison between the convergence of the aforementioned solvers when applied to the PML-terminated microstrip line shown in Figure 2. All three solvers were terminated when the residual error reached 0.01 or less. As seen, the GMRES solver has a significantly better error history.
GMRES employs the projection method [12] to solve the following linear system:

$$
\mathbf{A x}=\mathbf{b}
$$

where $\mathbf{A}$ is the system (FEM) matrix of size $(n \times n), \mathbf{x}$ is the solution vector of length $n$, and $\mathbf{b}$ is the feed (excitation) of the system. We seek an approximate solution $\mathbf{x}_{\mathbf{m}}$ from an affine subspace of reduced dimension $m$ (where $m \leq n$ ) [12]. This subspace is referred to as the Krylov subspace. Without going through the mathematical details, this minimization is based on finding a set of $m$ basis functions which span the solution space. At each iteration, the projections of $\mathbf{A}$ on all basis functions are evaluated, and $m$ steps of the Arnoldi modified Gram-Schmidt (MGS) procedure [12] are executed to obtain an approximate estimate of the solution, along with the basis functions ( $m$-vectors) for the next iteration. The GMRES iterations continue until convergence is reached.

Although the number of basis functions $m$ is arbitrary, it is the main parameter that affects solver convergence. In general, larger values of $m$ lead to smaller residuals, and hence faster convergence. The CPU and memory costs for GMRES are strongly dependent on $m$. The memory cost of the GMRES solver is $O(m n)$, while its computational complexity is $O\left(m^{2} n\right)$ [12]. Therefore, it is essential to have an approximate estimate for $m$ before executing the GMRES iterations [16]. If this estimate is too low, convergence will be extremely slow, and may not be achieved at all. On the other hand, if $m$ is too high, storage and CPU are wasted. The optimal value of $m$ is directly related to two main factors: the condition and size of the matrix, both of which affect convergence. Preconditioners are usually very effective in speeding up convergence (see, for example, [5]).

The key issue in the application of preconditioners is the balance between their CPU cost and the delivered improved convergence. Preconditioners vary in cost from the diagonal preconditioner (DPC), which has trivial implementation, to the complicated approximate inverses preconditioner (AIPC), 


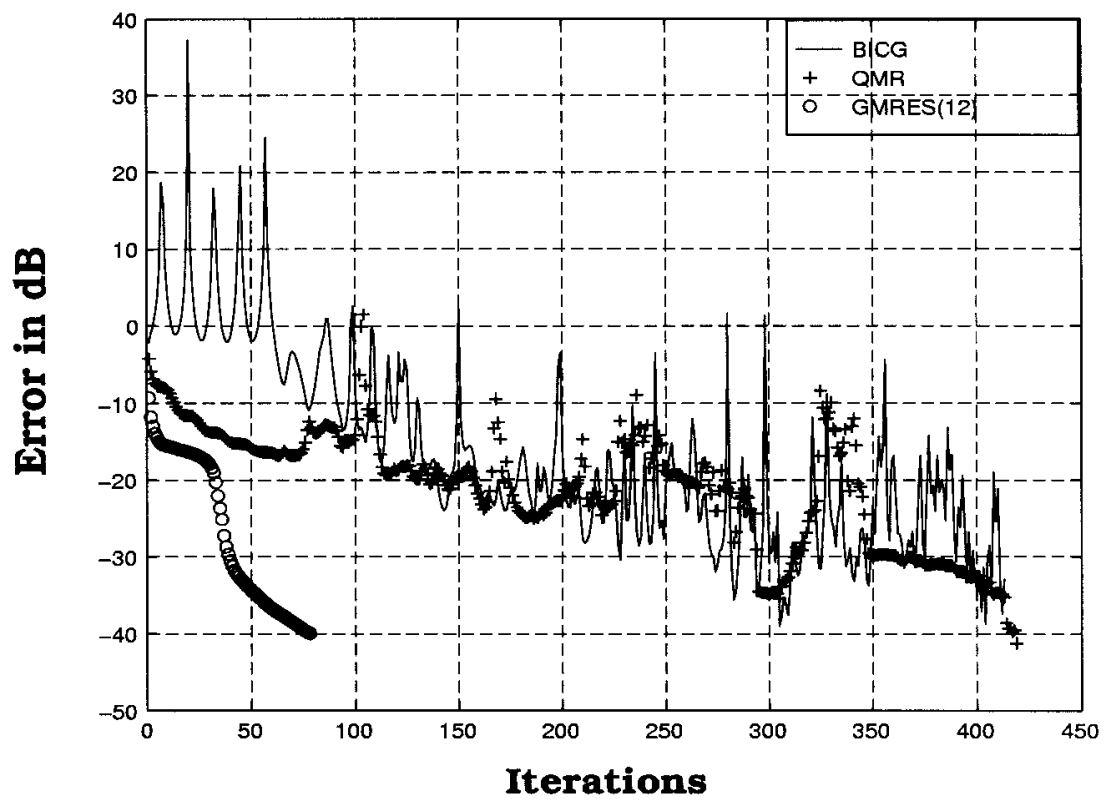

Figure 5 Comparison of the convergence among three different solvers

which results in full preconditioning matrices [8]. As shown in $[5,8]$, the AIPC within GMRES proved very successful when dealing with PML-terminated meshes. According to these studies, it was concluded that employing the AIPC achieves a significant reduction in the overall CPU time, in addition to removing the strong dependence on $m$. A pseudocode of the preconditioned GMRES is given in the Appendix.

\section{APPLICATIONS}

In this section, two representative applications are considered. The first deals with feed modeling in microwave circuits and filters. Specifically, we examine the minimum number of feed (current) probes required to maintain stable input impedance calculations. A spiral inductor with an air bridge is also considered as a more general application of PML absorbers. In both cases, the PML was implemented with the optimal values $\alpha=\beta=1$. The sampling rate was set to about 12-15 samples per linear wavelength. After performing the FEM simulation, total fields within the computational domain were obtained and decomposed to extract both incident and reflected fields at particular locations within the domain. The GMRES solver coupled with the AIPC [8] was applied to solve the resulting linear systems with 10-20 basis functions (search vectors).

5.1. Feed Analysis. Our goal in this application is to find the minimum number of probes required for a stable input impedance prediction for the microstrip line shown in Figure 2 , with the probes placed horizontally at the left starting point of the line. To do this, we fix all parameters of the circuit and absorber, and only change the number of feed probes. The number of probes is varied from one to four, and each time, the input impedance is computed and expressed as

$$
Z_{\text {in }}(N)=R_{\text {in }}(N)+j X_{\text {in }}(N)
$$

where $R_{\text {in }}(N)$ and $X_{\text {in }}(N)$ are the real and imaginary parts, respectively, of the input impedance when $N$ probes are employed to feed the line, and $j$ is the square root of -1 .
To characterize the accuracy of the calculated input impedance, two percentage error parameters are defined, namely,

$$
\delta_{r}(N)=\frac{\left|R_{\text {in }}(N)-R_{\text {in }}(N+1)\right|}{R_{\text {in }}(N+1)} \times 100
$$

and

$$
\delta_{i}(N)=\frac{\left|X_{\text {in }}(N)-X_{\text {in }}(N+1)\right|}{X_{\text {in }}(N+1)} \times 100
$$

for the real and imaginary parts, respectively. Figure 6 displays these parameters as a function of $N$. From this figure, the following can be deduced.

- The real part of the input impedance is fairly constant, and does not change much as the number of probes changes.

- The imaginary part of the input impedance is sensitive to the choice of the feed probes, and decreases as the number of probes increases.

- When the number of probes exceeds three, the value of the imaginary part converges, and therefore, three or more probes are sufficient for an accurate estimate of the input impedance from a finite-element simulation.

As seen in Figure 7, the number of probes has a minor effect on field distribution under the strip line. However, the input impedance of the circuit is strongly dependent on the feed mechanism. It can also be seen that field levels at a certain location within the computational domain increase linearly with the number of probes.

5.2. Spiral with an Air Bridge. The geometry of the spiral antenna with an air bridge is shown in Figure 8. For analysis, the spiral is placed in a cavity terminated with the PML absorber, and we should remark that this geometry has sufficient complexities to assure robust evaluation of the 


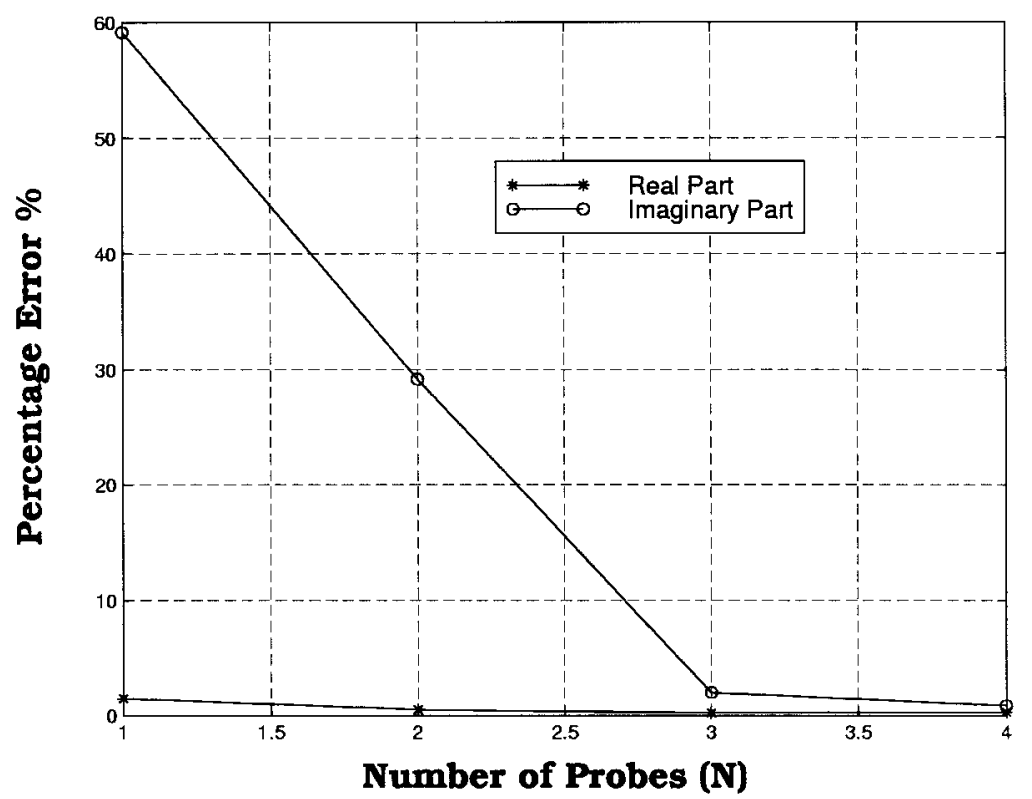

Figure 6 Variation of the microstrip-line input impedance for different numbers of feed probes used in the FEM model

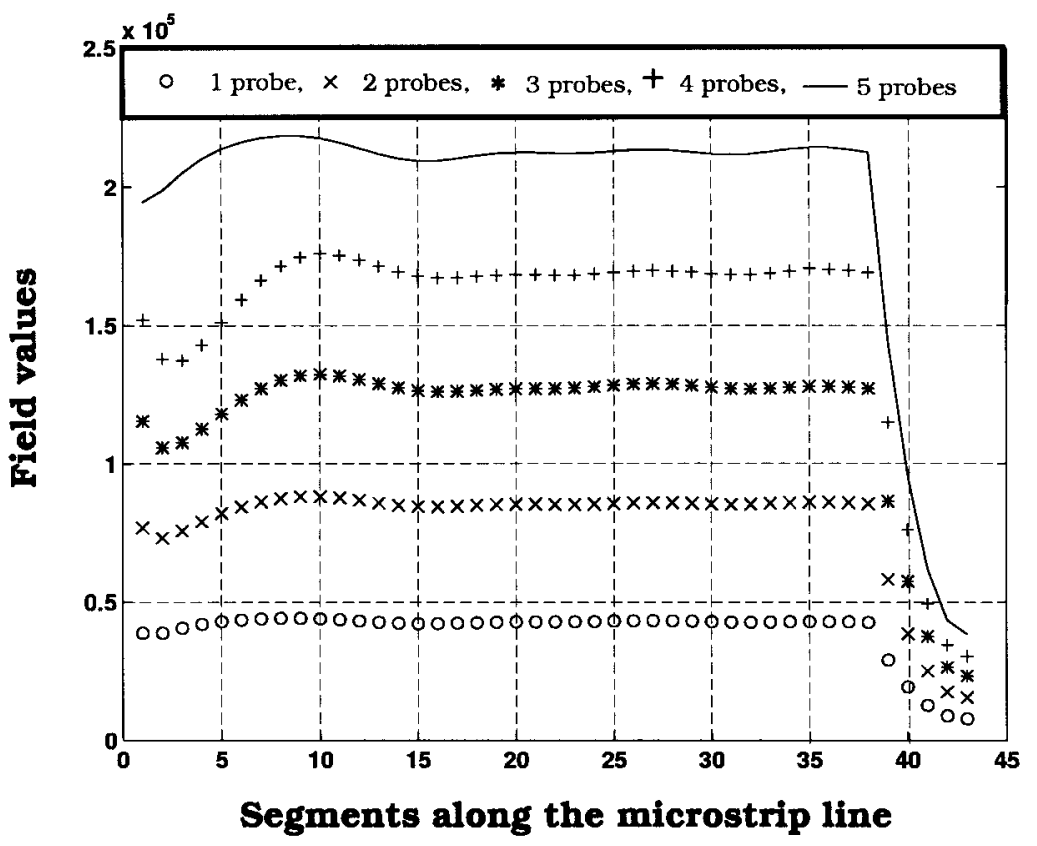

Figure 7 Field magnitude under the microstrip line for different numbers of feed probes used in the FEM model

PML termination. The computed scattering parameter magnitude $\left|S_{11}\right|$ is shown in Figure 9, and it is clear that good agreement exists between the measured and calculated data. To reduce the FEM system size, in our calculations, we assumed that the width of the air bridge equals that of the microstrip line. This is likely the cause of differences between the measured and computed data.

\section{CONCLUSIONS}

For large FEM systems, iterative solvers are used to alleviate memory and CPU demands. However, iterative solvers introduce convergence issues. Furthermore, PML absorbers deteriorate convergence even further. The main goal of this work was to speed up iterative solvers convergence of FEM systems when PML absorbers are used to truncate the mesh. An important aspect of our work was the optimal selection of the PML parameters so that absorption is maximized without further deterioration in convergence. Using these parameters, the generalized minimal residual (GMRES) solver was tested for two microwave circuit applications. Preconditioning does, of course, lead to convergence speedup, and the reader is referred to $[5,8,14]$ for more on this subject.

\section{APPENDIX}

The pseudocode of the preconditioned GMRES algorithm is as follows [14]. 


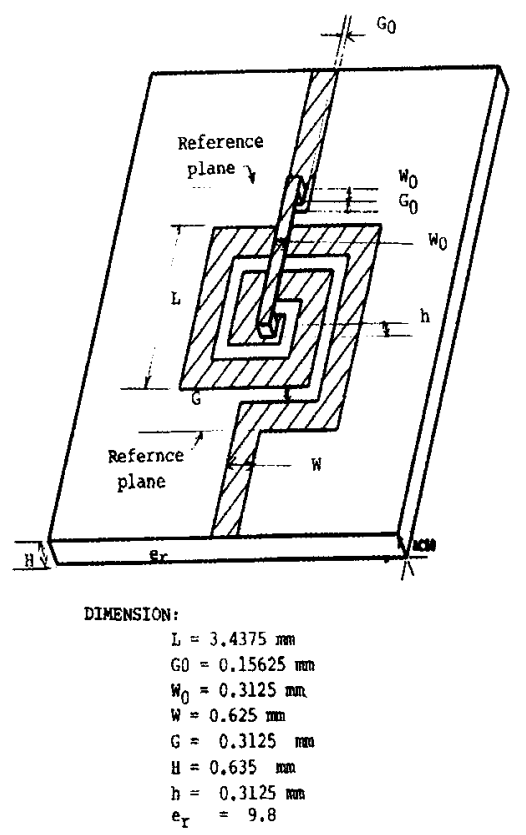

Figure 8 Geometry of the spiral with an air bridge

Initialize $\mathbf{x}$

$\mathbf{r}_{\mathbf{0}}=\mathbf{b}-\mathbf{A x}$

resd $=\sqrt{\mathbf{r}_{0}^{*} \cdot \mathbf{r}_{0}}$

$\mathbf{v}_{\mathbf{1}}=\mathbf{r}_{\mathbf{0}} /$ resd (forming the basis functions).

Define the $m+1 \times m$ matrix $\mathbf{H}_{\mathbf{m}}=\mathbf{h}_{\mathbf{i j}}, 1 \leq i \leq m+1$ and $1 \leq j \leq m$ (Hessenberg matrix [12]).

Set $\mathbf{H}_{\mathbf{m}}=\mathbf{0}$.

For $j=1,2, \ldots, m$ Do

$\mathbf{w}_{\mathbf{j}}=\mathbf{A M} \mathbf{v}_{\mathbf{j}}, \mathbf{M}$ is the preconditioner.
For $i=1,2, \ldots$ Do

$$
\begin{aligned}
& \mathbf{h}_{\mathbf{i}, \mathbf{j}}=\sqrt{\mathbf{w}_{\mathbf{j}}^{*} \cdot \mathbf{v}_{\mathbf{j}}} \\
& \mathbf{w}_{\mathbf{j}}=\mathbf{w}_{\mathbf{j}}-\mathbf{h}_{\mathbf{i}, \mathbf{j}} \mathbf{v}_{\mathbf{i}} . \\
& \text { End } \\
& \mathbf{h}_{\mathbf{j}+\mathbf{1}, \mathbf{j}}=\sqrt{\mathbf{w}_{\mathbf{j}}^{*} \cdot \mathbf{w}_{\mathbf{j}}} \\
& \mathbf{v}_{\mathbf{j}+\mathbf{1}}=\mathbf{w}_{\mathbf{j}} / \mathbf{h}_{\mathbf{j}+\mathbf{1}, \mathbf{j}} \\
& \text { End }
\end{aligned}
$$

Compute $\mathbf{y}_{\mathbf{m}}$, the minimizer of $\sqrt{\mathbf{s}^{*} \cdot \mathbf{s}}$, where $\mathbf{s}=\operatorname{resd} * \mathbf{e}_{\mathbf{1}}$ $-\mathbf{H} * \mathbf{y}$ and $\mathbf{e}_{\mathbf{1}}=\left[\begin{array}{llll}\mathbf{1} & 0 & 0 & \ldots\end{array}\right]^{T} \mathbf{x}=\mathbf{x}+\mathbf{M V}_{\mathbf{m}} \mathbf{y}_{\mathbf{m}}$

Check convergence; if achieved, stop iterations; else, restart iterating.

\section{REFERENCES}

1. Z.S. Sacks, D.M. Kingsland, R. Lee, and J.F. Lee, A perfectly matched anisotropic absorber for use as an absorbing boundary condition, IEEE Trans Antennas Propagat (1994).

2. J.P. Berenger, A perfectly matched layer for the absorption of electromagnetic waves, J Comput Phys 114 (1994), 185-2000.

3. B. Engquist and A. Majda, Absorbing boundary conditions for the numerical simulation of waves, Math Comput 31 (1977), 629-651.

4. T.B.A. Senior and J.L. Volakis, Approximate boundary conditions in electromagnetics, IEE Press, Stevenage, England, 1995, chap. 8.

5. Y.Y. Botros and J.L. Volakis, Detailed convergence study for perfectly matched layer (PML) applications, 14th Appl Computational Electromag (ACES) Conf, Naval Postgraduate School, Monterey, CA, Mar. 1998.

6. W.C. Chew and W.H. Weedon, A 3-D perfectly matched medium from modified Maxwell's equations with stretched coordinates, Microwave Opt Technol Lett (Sept. 1994), 599-604.

7. A.C. Polycarpou and C.A. Balanis, An optimized anisotropic PML for the analysis of microwave circuits, IEEE Microwave Guided Wave Lett (Feb. 1998), 30-32.

8. Y.Y. Botros and J.L. Volakis, Preconditioned generalized minimal residual iterative scheme for perfectly matched layer terminated applications, IEEE Microwave Guided Wave Lett (accepted).

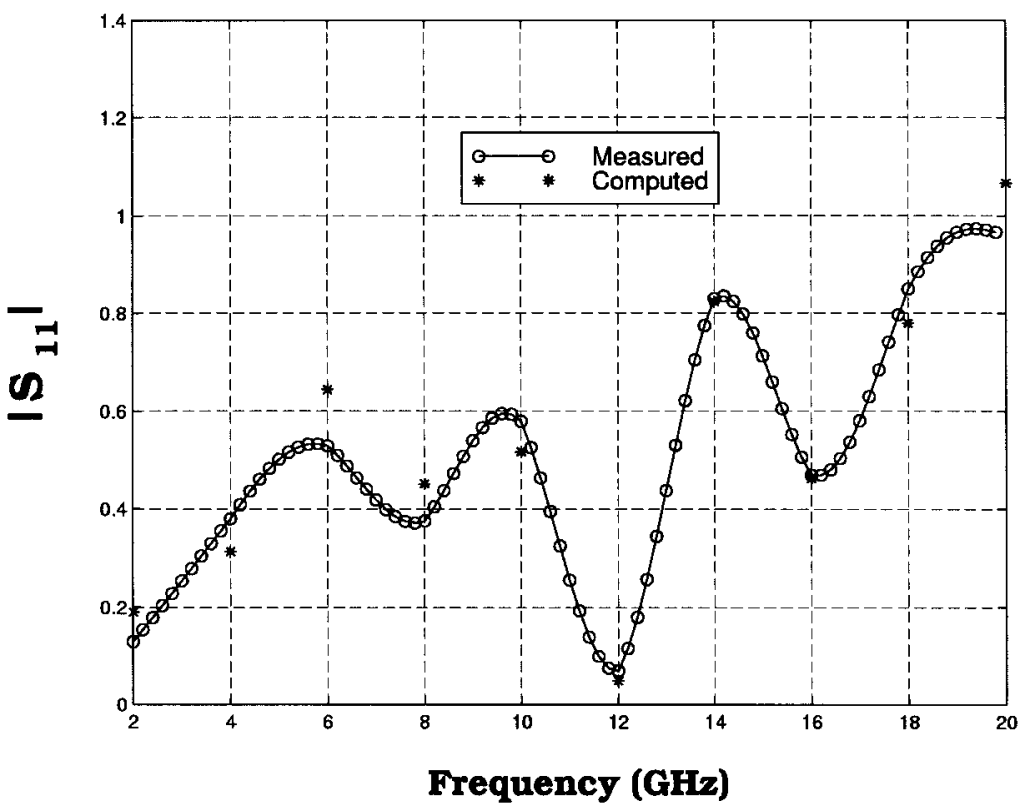

Figure 9 Comparison between the measured and computed data for the spiral inductor shown in Figure 8 
9. S. Legault, T.B.A. Senior, and J.L. Volakis, Design of planar absorbing layers for domain truncation in FEM applications, Electromag J 16 (1996).

10. D.M. Kingsland, J. Gong, J.L. Volakis, and J.F. Lee, Performance of an anisotropic artificial absorber for truncating finite element meshes, IEEE Trans Antennas Propagat (Dec. 1995).

11. J.F. Lee, Tangential vector finite element methods and their application to solving EM scattering problems, ACES Newsletter (Mar. 1995), invited tutorial paper, (nonrefereed).

12. Y. Saad, Iterative methods for sparse linear system, PWS Publishing, 1996.

13. R. Barret et al., Templates for the solution of linear systems: Building blocks for iterative solvers, SIAM, 1994.

14. J.L. Volakis, A. Chatterjee, and L.C. Kempel, Finite element method for electromagnetics, IEEE Press, New York, 1998.

15. G.H. Golub and C.F. Van Loan, Matrix computations, The Johns Hopkins University Press, Baltimore, MD, 1985.

16. A.D. Brown, J.L. Volakis, L.C. Kempel, and Y.Y. Botros, Patch antennas on ferromagnetic substrates, IEEE Trans Antennas Propagat (to appear).

(c) 1999 John Wiley \& Sons, Inc.

CCC 0895-2477/99

\section{EXTENSION AND VALIDATION OF EQUIVALENT SOURCE HELICAL ANTENNA MODELING WITH THE FDTD CODE}

\author{
Gianluca Lazzi, ${ }^{1}$ Qi-Shan Yu, ${ }^{1}$ and Om P. Gandhi ${ }^{1}$ \\ ${ }^{1}$ Department of Electrical Engineering \\ University of Utah \\ Salt Lake City, Utah 84112-9206
}

Received 12 May 1999

\begin{abstract}
Since small-diameter, normal-mode helical antennas are often used for cellular telephones, accurate modeling of these antennas using the FDTD grid is a must if this preferred method, allowing anatomically based heterogeneous simulation of the body, is to be used for calculating the performance of personal wireless devices. An easy-touse rectangular FDTD grid is, however, subject to staircasing for the helical geometry. An approach using equivalent electric and magnetic sources has previously been presented by the authors, although not thoroughly tested for cases where the diameter of the helix may be more than two times the FDTD grid size. In this letter, we present a modified weighted equivalent source method and its experimental validation for such larger diameter helical antennas. The grid is selected to conform to the overall dimensions of the helix. The calculated field variations for a lossy sphere representative of the human head agree well with the measured data. (c) 1999 John Wiley \& Sons, Inc. Microwave Opt Technol Lett 23: 172-174, 1999.
\end{abstract}

Key words: helical antennas; SAR; FDTD

\section{INTRODUCTION}

The finite-difference time-domain (FDTD) method [1] is widely used for the solution of bioelectromagnetic problems since it allows use of the heterogeneous, anatomically based models of the exposed objects. Several authors have therefore used the FDTD method to evaluate the rates of energy deposition (specific absorption rate or SAR) induced in the human head by cellular telephones [2-5]. A concern is that the antenna be appropriately modeled to reproduce its radiation characteristics accurately. Helical antennas, or antennas involving helix-whip combinations, are extremely popular for cellular telephones today. A major problem for cases where the helical antenna needs to be modeled arises from the staircase approximation of the antenna geometry with the FDTD grid. Such an approximation may lead to errors that are difficult to estimate, and other methods therefore need to be considered. An obvious solution consists of the use of the subgridding technique [6] to model the antenna with a finer resolution to reduce the staircasing effect, but this considerably complicates the FDTD code, and reduces its efficiency in terms of memory and time requirements for computation.

In [7], we have introduced a new method to model smalldiameter normal-mode helical antennas with the FDTD code for cellular telephone applications. A question arises as to whether such an approach using equivalent electric- and magnetic-field-based sources can also be used for somewhat larger diameter helical antennas, such as the one suggested by the IEEE Standards Coordinating Committee SCC-34 as a benchmark test to validate the modeling capabilities of the FDTD code for complex structures. The specific test involves the use of a $7.82 \mathrm{~mm}$ diameter $835 \mathrm{MHz}$ helical dipole placed very close (including physical contact) to a glass sphere filled with a lossy, high-dielectric-constant, brain simulant material. The diameter of the helical dipole, being considerably larger than the FDTD cell size, required modifications in the formulation of the equivalent sources as compared to those presented in [7]. The procedure described in this letter gives results that are in good agreement with measurements for SAR in the experimental model.

\section{GEOMETRY OF THE PROBLEM AND THE FDTD MODELING}

The geometry of the considered problem is shown in Figure 1 , where the important dimensions are indicated. The glass sphere has an outer diameter of $11 \mathrm{~cm}$, glass thickness of $5 \mathrm{~mm}$, and the dielectric constant of the glass is $\epsilon_{r}=4$. The liquid filling the sphere is made of a composition of water, salt, and sugar, and its dielectric properties represent the average for white and gray matter of the human brain $\left(\epsilon_{r}=42.8, \sigma=1.125 \mathrm{~S} / \mathrm{m}\right.$.) As shown in Figure 1, the helical dipole is fed by means of a balun. The total length of the helix is $52.5 \mathrm{~mm}$, its diameter is $7.82 \mathrm{~mm}$, and the pitch is $2.45 \mathrm{~mm}$. The SAR induced in the sphere for three distances $d(0,5$, and $25 \mathrm{~mm})$ between the helix and the outer edge of the sphere was required. To have a reasonable-size FDTD space, we have chosen to use a cubical cell size of $2.5 \mathrm{~mm}$. Being a near-field problem, the helical dipole needs to be represented with a size (cross section and length) close to the physical size of the antenna. Therefore, the whole helical dipole has been modeled by means of a stack of 21 layers of nine cells $(3 \times 3)$ for each of the cross sections. To model the cross section of the helical dipole by means of nine cells, we have extended the concept introduced in [7] that uses electric and magnetic sources for each cross section of the helix. The relative electric field as calculated by means of the equations given in [7] should be divided among all of the cells in each cross-sectional plane chosen to represent the helix (see Fig. 2). If a corner is common to four cells, the value of the electric field in that location is set to be four times the value assumed in a corner belonging to just one cell. Similarly, if a corner is common to two cells, the value of the electric field will be twice the value assumed in a corner belonging to a single cell. After calculating the relative weights of electric and magnetic sources, according to the equations given in [7], 